\title{
RELATIVE $K$-CYCLES AND ELLIPTIC BOUNDARY CONDITIONS
}

\author{
GUIHUA GONG
}

Dedicated to Professor Zejian Jiang on his seventieth birthday

\begin{abstract}
In this paper, we discuss the following conjecture raised by BaumDouglas: For any first-order elliptic differential operator $D$ on smooth manifold $M$ with boundary $\partial M, D$ possesses an elliptic boundary condition if and only if $\partial[D]=0$ in $K_{1}(\partial M)$, where $[D]$ is the relative $K$-cycle in $K_{0}(M, \partial M)$ corresponding to $D$. We prove the "if" part of this conjecture for $\operatorname{dim}(M) \neq$ $4,5,6,7$ and the "only if" part of the conjecture for arbitrary dimension.
\end{abstract}

First we fix some notation. $M$ is a compact oriented smooth manifold with smooth boundary $\partial M$. We always suppose that $M$ is embedded in some compact smooth manifold $\widetilde{M}$ without boundary of the same dimension (e.g., $\widetilde{M}$ can be taken as double of $M)$. We denote $\stackrel{\circ}{M}=M \backslash \partial M$. Furthermore, we assume that $E_{0}$ and $E_{1}$ (in fact, all the vector bundles in this paper) are smooth complex Hermitian vector bundles over $M$ and that $D: C^{\infty}\left(E_{0}\right) \rightarrow C^{\infty}\left(E_{1}\right)$ is a first-order elliptic differential operator from smooth sections of $E_{0}$ to that of $E_{1}$. By $H^{s}\left(M, E_{i}\right)$ and $H^{s}\left(\partial M, E_{i}\right)$ we shall denote the Sobolev spaces of sections of $E_{i}$ and $\left.E_{i}\right|_{\partial M}$ with respect to fixed smooth measures on $M$ and $\partial M$, respectively.

The elliptic boundary value problem (an elliptic operator with an elliptic boundary condition) has been studied for a long time. As noted in $[1,5,6]$ and other references, there exist topological obstructions to impose an elliptic boundary condition on the above $D$. A fundamental problem is to find all such obstructions. Baum, Douglas, and Taylor constructed a relative K-cycle $[D] \in K_{0}(M, \partial M) \cong K K\left(C_{0}(\stackrel{\circ}{M}), \mathbb{C}\right)$ (here $C_{0}(\stackrel{\circ}{M})$ is the algebra of continuous functions on $M$ which vanish on $\partial M$ ) corresponding to $D$ (see [2-4] for details). From the definition of relative $\mathrm{K}$-homology group $K_{0}(M, \partial M)$ given by Baum, Douglas, and Taylor, the boundary map $\partial: K_{0}(M, \partial M) \longrightarrow K_{1}(\partial M)$ is very concrete [2-4]. Also Baum and Douglas conjectured that the only obstruction for $D$ possessing elliptic boundary conditions is that $\partial[D] \neq 0$. More precisely, the following conjecture first appeared in [2] in a closely related form.

Conjecture. There exist a vector bundle $E_{2}$ over $\partial M$ and a zeroth-order pseudodifferential operator $B$ defined from $C^{\infty}\left(\partial M, E_{0}\right)$ to $C^{\infty}\left(\partial M, E_{2}\right)$ such that

$$
\left(\begin{array}{c}
D \\
B \circ \gamma
\end{array}\right): H^{1}\left(M, E_{0}\right) \longrightarrow \begin{gathered}
H^{0}\left(M, E_{1}\right) \\
H^{1 / 2}\left(\partial M, E_{2}\right)
\end{gathered}
$$

Received by the editors March 24, 1992 and, in revised form, June 25, 1992.

1991 Mathematics Subject Classification. Primary 46L80, 46M20, 19K33, 35S15, 35G15. 
is Fredholm if and only if $\partial[D]=0$ in $K_{1}(\partial M)$, where $\gamma: H^{1}\left(M, E_{0}\right) \longrightarrow$ $H^{1 / 2}\left(\partial M, E_{0}\right)$ is the trace map.

Remark 1. Let $D$ be as above with pincipal symbol $p(x, \xi)$. A zeroth-order pseudo-differential operator $B$ with principal symbol $b(x, \xi)$ from $C^{\infty}\left(\partial M, E_{0}\right)$ to $C^{\infty}\left(\partial M, E_{2}\right)$ is said to be elliptic to $D$ (see [6], p. 233) if, for every $x \in \partial M$ and $\xi \in T_{x}^{*}(\partial M)$, the map $M_{x, \xi}^{+} \ni u \longrightarrow b(x, \xi) u(0) \in\left(E_{2}\right)_{x}$ is bijective, where $T_{x}^{*}(\partial M)$ and $\left(E_{2}\right)_{x}$ are the fibres at the point $x$ of the cotangent bundle $T^{*} \partial M$ and the bundle $E_{2}$, and, furthermore, $M_{x, \xi}^{+}$is the set of all $u \in C^{\infty}\left(\mathbb{R},\left(E_{0}\right)_{t}\right)$ with $p\left(x, \xi-i \frac{d}{d t} \cdot n_{x}\right) u(t)=0 \quad\left(n_{x}\right.$ is the interior conormal vector of $M$ at $x$ ) which are bounded on $\mathbb{R}_{+}$. If $B$ is elliptic to $D$, then the above $\left(\begin{array}{c}D \\ B \circ \gamma\end{array}\right)$ is Fredholm. Such a system $\left(\begin{array}{c}D \\ B \circ \gamma\end{array}\right)$ is often called an elliptic boundary value problem or an elliptic operator with an elliptic boundary condition; meanwhile, $D$ is also said to possess an elliptic boundary condition.

Remark 2. Although the above elliptic boundary condition is used in most references, the original form of the conjecture in [2] is in a slightly different form from the above. In [2] the operator for the boundary condition is of the form $\gamma \circ B$, where $B$ is a zeroth-order pseudo-differential operator from $E_{0}$ to a smooth vector bundle over a neighborhood of $M$. The reason we use a slightly different form of the conjecture is as follows: for general zeroth-order pseudodifferential operator $B$ defined on $\widetilde{M}$, there is no canonical way to restrict $B$ to $M$ as an operator $B_{M}: H^{s}(M) \longrightarrow H^{s}(M)$ when $s>0$. So one needs to put some restriction on $B$. One of the natural restrictions is that $B$ has the transmission property with respect to $\partial M$ (see [5]). We also prove our theorem for this kind of boundary condition (see Theorem 1). It must be pointed out that the existence of a boundary condition of type $B \circ \gamma$ implies the existence of that of type $\gamma \circ B$.

In this paper, we prove the "only if" part of the conjecture which can be thought of as a generalization of Corollary 4.2 in [4] (there $B$ is a differential operator). Conversely, we prove that if $\operatorname{dim}(M) \neq 4,5,6,7$ and $\partial[D]=0$, then $D$ possesses an elliptic boundary condition as in Remark 1. Hence the "if" part of the conjecture has been proved for $\operatorname{dim}(M) \neq 4,5,6,7$. The cases of $\operatorname{dim}(M)$ being equal to $4,5,6$, or 7 are still open, but we prove a theorem which can be thought of as the "if" part of the conjecture in the sense of stablization in K-homology group for arbitrary dimension. Our results will be useful for constructing absolute $K$-cycles in $K_{0}(M)$ which are preimages of $[D] \in K_{0}(M, \partial M)$ under the canonical map from $K_{0}(M)$ to $K_{0}(M, \partial M)$ when $\partial[D]=0$.

Our main results are the following:

Theorem 1. ("only if " part) $\partial[D]=0$ if one of the following is true:

(i) There exist a smooth vector bundle $E_{2}$ over $\partial M$ and a zeroth-order pseudodifferential operator $B$ from $\left.E_{0}\right|_{\partial M}$ to $E_{2}$ such that $\left(\begin{array}{c}D \\ B \circ \gamma\end{array}\right)$ in the conjecture is Fredholm.

(ii) There exist a bundle $E_{2}$ over a neighborhood of $M$ in $\widetilde{M}$ and a zerothorder pseudo-differential operator $B$ with transmission property with respect to 
$\partial M$ from $E_{0}$ to $E_{2}$ such that

$$
\left(\begin{array}{c}
D \\
\gamma \circ B
\end{array}\right): H^{1}\left(M, E_{0}\right) \longrightarrow \begin{gathered}
H^{0}\left(M, E_{1}\right) \\
H^{1 / 2}\left(\partial M, E_{2}\right)
\end{gathered}
$$

is Fredholm.

Theorem 2. ("if" part) If $\partial[D]=0$, then there exists a first-order elliptic differential operator $D_{1}$ acting on smooth vector bundles over $M$ with $\left[D_{1}\right]=0$ in $K_{0}(M, \partial M)$ such that $D \oplus D_{1}$ possesses an elliptic boundary condition as in Remark 1, and, furthermore, if $\operatorname{dim}(M) \neq 4,5,6,7$, then $D$ itself possesses an elliptic boundary condition.

The main idea of the proof of Theorem 1 is to construct an intertwining between $\partial[D]$ and a trivial element in $K_{1}(\partial M)$. In the proof, we use Calderon projection, functional calculus of pseudo-differential operators (including Boutet de Monvel type operators), and the techniques in the proof of Proposition 4.5 of [4].

The proof of Theorem 2 makes use of two key lemmas (see below).

Let $S T^{*} \partial M$ be the unit sphere bundle of $T^{*} \partial M$ over $\partial M$ and $\pi$ : $S T^{*} \partial M \longrightarrow \partial M$ be the canonical projection map. Let $\widetilde{E}_{0}=\pi^{*}\left(\left.E_{0}\right|_{\partial M}\right)$ be the bundle over $S T^{*} \partial M$. We write the principal symbol of $D$, in a coordinate neighborhood $U$ of $x \in \partial M$, as

$$
p\left(x, x_{n}, \xi, \xi_{n}\right)=\sum_{j=1}^{n-1} p_{j}\left(x, x_{n}\right) \xi_{j}+p_{n}\left(x, x_{n}\right) \xi_{n},
$$

where $x_{n}$ is the coordinate for the normal direction of $\partial M$. We define

$$
\tau(x, \xi)=i p_{n}^{-1}(x, 0) \sum_{j=1}^{n-1} p_{j}(x, 0) \xi_{j}
$$

for $x \in \partial M$ and $\xi \in S T^{*} \partial M$. Then $\tau(x, \xi)$ is a map from a fibre of $E_{0}$ into itself and has no purely imaginary eigenvalue. Let $V_{ \pm}$be the subbundle of $\widetilde{E}_{0}$ over $S T^{*} \partial M$ corresponding to the span of the generalized eigenvectors of $\tau(x, \xi)$ corresponding to the eigenvalues with positive/negative real parts.

Lemma 1. $\partial[D]=0$ if and only if $\left[V_{+}\right] \in \pi^{*} K^{0}(\partial M) \subset K^{0}\left(S T^{*} \partial M\right)$.

Lemma 2. If $E_{0}$ and $E_{1}$ are vector bundles over $M$ which allow a first-order elliptic differential operator $D$ to act from one to the other, and if $\operatorname{dim}(M)=n$, then

(i) $f \operatorname{dim}\left(E_{0}\right)=f \operatorname{dim} E_{1} \geq 2^{[(n-1) / 2]}$;

(ii) $f \operatorname{dim}\left(E_{0}\right)=f \operatorname{dim} E_{1} \geq 2^{[(n-1) / 2]+1}$ provided $n$ is even and $\partial[D]=0$,

where $f$ dim denotes dimension of each fibre of the vector bundles.

The Proof of Theorem 2. By Lemma 1, if $\partial[D]=0$, one has

$$
\left[V_{+}\right] \in \pi^{*} K^{0}(\partial M) \subset K^{0}\left(S T^{*} \partial M\right) .
$$

By Lemma 2, $f \operatorname{dim}\left(V_{+}\right)=\frac{f \operatorname{dim}\left(E_{0}\right)}{2} \geq 2^{[n / 2]-1}$. Therefore, $f \operatorname{dim}\left(V_{+}\right) \geq n-1>$ $\frac{\operatorname{dim}\left(S T^{*} \partial M\right)}{2}=\frac{2 n-3}{2}$, whenever $\operatorname{dim}(M) \geq 8$. Hence there exists a complex 
vector bundle $E_{2}$ over $\partial M$, such that $V_{+} \cong \pi^{*} E_{2}$. This is also true for $\operatorname{dim}(M) \leq 3$, since the collection of complex vector bundles over $S T^{*} \partial M$ $\left(\operatorname{dim}\left(S T^{*} \partial M\right) \leq 3\right)$ has property of cancellation.

Let $\psi$ be the bundle isomorphism

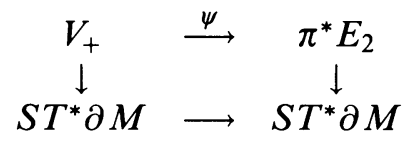

For any $(x, \xi) \in S T^{*} \partial M$, let $b(x, \xi)$ be the bundle map defined by

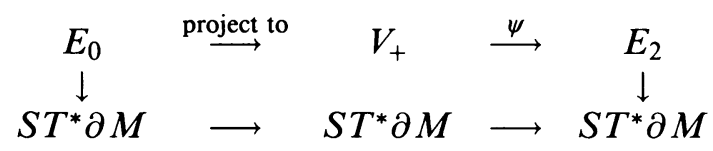

Furthermore, let $B:\left.E_{0}\right|_{\partial M} \longrightarrow E_{2}$ be the zeroth-order pseudo-differential operator with symbol $b(x, \xi)$. It follows that $B$ is elliptic to $D$ (see [6]).

Example 1. If $M$ is a $\operatorname{spin}^{c}$ manifold with smooth boundary and $D$ is the Dirac operator over $M$, then it is computed in [4] that $\partial[D] \neq 0$. Hence $D$ possesses no elliptic boundary condition (even possesses no boundary condition as in the conjecture).

Example 2. For any $D$, let $D^{*}$ be the formal adjoint of $D$. It is easy to prove that $[D]=-\left[D^{*}\right]$ in $K_{0}(M, \partial M)$; hence, $\partial\left[D \oplus D^{*}\right]=0$. It follows that $D \oplus D^{*}$ possesses an elliptic boundary condition provided $\operatorname{dim}(M) \neq 4$ or 6 . (It should be noted that we only need to exclude the manifolds with dimension 4 and 6 here, since the dimension of the bundle on which $D \oplus D^{*}$ acts is twice the dimension of the bundle on which $D$ acts.)

The details of the proofs will appear elsewhere.

\section{ACKNOWLEDGMENTS}

This work was done while the author was a postdoctoral fellow at the University of Toronto. He is grateful to Professors George A. Elliott and Man-Deun Choi for their support. The author also thanks Professors Man-Deun Choi, Ronald G. Douglas, George A. Elliott, and Peter Greiner and Ms. Liangqing Li for many helpful conversations.

\section{REFERENCES}

1. M. Atiyah, V. Patodi, and I. Singer, Spectral asymmetry and riemannian geometry. I, II, III, Math. Proc. Cambridge Philos. Soc. 77 (1975), 43-69; 78 (1975), 405-632; 79 (1976), 71-99.

2. P. Baum and R. Douglas, Index theory, bordism, and K-homology, Operator Algebras and $K$-Theory (R. G. Douglas and C. Schochet, eds.), Contemp. Math., vol. 10, Amer. Math. Soc., Providence, RI, 1982, pp. 1-31.

3. _ Relative $K$-homology and $C^{*}$-algebra, manuscript.

4. P. Baum, R. Douglas, and M. Taylor, Cycles and relative cycles in analytic $K$-homology, J. Differential Geom. 30 (1989), 761-804. 
5. L. Boutet de Monvel, Boundary problems for pseudodifferential operators, Acta Math. 126 (1971), 11-51.

6. L. Hömander, The analysis of linear partial differential operators. III, Springer, New York, 1985.

Department of Mathematics, University of Toronto, Toronto, Ontario, Canada M5S 1A1, and Department of Mathematics, Jilin University, People's Republic of China

Current address: Department of Mathematics, Queen's University, Kingston, Canada K7L 3N6

E-mail address: guihua@math.toronto.edu 